\title{
Estructura y dinámica de bosques de palo santo en el Chaco Seco
}

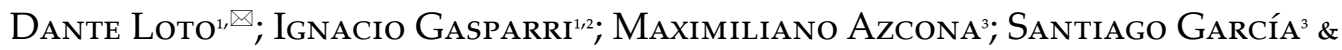 \\ Carlos SpagarinO
}

\begin{abstract}
${ }^{1}$ Facultad de Ciencias Naturales e IML. Universidad Nacional de Tucumán. Tucumán, Argentina. ${ }^{2}$ Instituto de Ecología Regional. Facultad de Ciencias Naturales e IML. Universidad Nacional de Tucumán. Tucumán, Argentina. ${ }^{3}$ Dirección de Bosques. Subsecretaría de Planificación y Ordenamiento Ambiental del Territorio. Ministerio de Ambiente y Desarrollo Sustentable de la Nación, C.A.B.A., Argentina. ${ }^{4}$ Departamento de Conservación y Uso Público. Parque Nacional Río Pilcomayo. Laguna Blanca. Formosa, Argentina.
\end{abstract}

\begin{abstract}
Resumen. El conocimiento de la estructura y la dinámica de las poblaciones de Bulnesia sarmientoi ("palo santo") es necesario para una gestión forestal capaz de conservar la especie, asegurar la producción maderera de alta calidad y preservar los servicios ecosistémicos que brindan los bosques donde se encuentra. Se caracterizó la estructura y la dinámica forestal en bosques con presencia de palo santo en el norte del Chaco Seco argentino. Se establecieron 21 conglomerados compuestos cada uno por cuatro juegos de parcelas concéntricas de $1000 \mathrm{~m}^{2}$ (diámetro $>20 \mathrm{~cm}$ ) y $500 \mathrm{~m}^{2}$ (diámetro $>10 \mathrm{~cm}$ ), ubicadas en los vértices de un cuadrado de $100 \mathrm{~m}$ de lado. Los conglomerados se midieron en 2007 y 2012. Se realizaron cálculos de parámetros estructurales y del índice de valor de importancia de cada especie en cada conglomerado. Se hallaron tres estructuras típicas: a) palosantales de bañado, b) palo cruz con palo santo, y c) bosque chaqueño con presencia de palo santo. A partir de la remedición de los árboles de palo santo se calculó un incremento periódico anual de $1.14 \mathrm{~mm} /$ año. Tomando como referencia la estructura de un conglomerado con palo santo que fue aprovechado entre mediciones se realizaron simulaciones de intervenciones alternativas (remoción de $80 \%$ de individuos de todas las clases diamétricas o aplicación de restricción de diámetro $>40 \mathrm{~cm}$ ) sobre la dinámica del rodal. Las proyecciones a 50 años muestran que la corta de $80 \%$ es muy severa, lo que indicaría que los aprovechamientos de tamaños menores para postes pueden comprometer la recuperación del rodal. En las cortas centradas en los tamaños mayores se lograría una recuperación en 50 años. En función de nuestros resultados, se sugiere un enfoque de uso de la especie centrado en productos de gran tamaño y con el mayor valor agregado posible.
\end{abstract}

[Palabras clave: Bulnesia sarmientoi, crecimiento, simulación de cortas, dinámica de rodal]

\begin{abstract}
Aвstract. Structure and dynamics of palo santo forests in the Dry Chaco. Knowledge on the structure and dynamics of Bulnesia sarmientoi ("palo santo") populations is necessary for forest management aimed at the conservation and high-quality timber production by this species and the preservation of the ecosystem services of its forests. We characterized forest structure and dynamics in stands with palo santo presence in the Nothern Argentinian Dry Chaco. We sampled 21 forest sampling clusters. Each forest sampling cluster includes four sets of concentric circular plots of $1000 \mathrm{~m}^{2}$ (diameter $\left.>20 \mathrm{~cm}\right)$ and $500 \mathrm{~m}^{2}$ (diameter $>10 \mathrm{~cm}$ ), located at the vertices of a square of $100 \mathrm{~m}$ side. Forest clusters were measured in 2007 and 2012. We calculated the importance value index (IVI) of each species in each conglomerate. We identified three typical structures with palo santo: a) palosantales in regularly flooded soils, b) palo cruz con palo santo, and c) Chaco mixed forest with the presence of palo santo. Periodic annual increment in diameter was $1.14 \mathrm{~mm}$ for all diameter classes. Taking as a reference the structure of a conglomerate with palo santo that was selective logged, we simulated alternative interventions ( $80 \%$ removal of all diameter classes or a diameter restriction of $>40 \mathrm{~cm}$ ) on stand dynamics. Cutting of $80 \%$ of the trees was a very severe intervention, suggesting that the removal of the smallest sizes for rural construction could compromise the recovery of the stand. A minimum size restriction could warrant recovery in 50 years. We suggest that the harvest of palo santo trees should be focused on large individuals with the highest added value potential.
\end{abstract}

[Keywords: Bulnesia sarmientoi, growth, logging simulations, forest stand dynamics]

\section{INTRODUCCIÓN}

La presión por nuevas pasturas y tierras agrícolas sostiene altas tasas de deforestación en áreas tropicales y subtropicales (Hansen et al. 2013). Además, gran parte de los bosques naturales tropicales se usan de manera regular para cortas selectivas de maderas de alto valor, lo cual, en muchas ocasiones, deja las

Editor asociado: Guillermo Martínez Pastur

凶 danteloto87@gmail.com áreas aprovechadas vulnerables a procesos tales como incendios, cacería o deforestación (Edwards et al. 2014). Administrar el uso de los recursos con alto valor maderero es un tema central de discusión en el marco de una creciente demanda de especies de maderas valiosas en el mercado global. A la vez, también se busca conservar la biodiversidad

Recibido: 23 de junio de 2017

Aceptado: 22 de noviembre de 2017 
y los servicios ecosistémicos (CITES 2012), por lo cual el concepto de degradación de bosques se vuelve central para iniciativas como la reducción de las emisiones de carbono por deforestación y degradación de bosques (REDD+) (Sasaki and Putz 2009). Un primer paso necesario para manejar de forma más planificada los recursos forestales es conocer los aspectos básicos de la estructura y la dinámica forestal del bosque con especies valiosas, a fin de determinar el grado de resiliencia de las comunidades forestales afectadas. La estructura forestal es la distribución de árboles en una superficie determinada, mientras que la dinámica representa el cambio de dicha estructura con el tiempo (Lamprecht 1990; Oliver and Larson 1996). Disponer de referencias de estructura y dinámica del bosque es útil, ya que permite usar, como primera aproximación, modelos sencillos para evaluar tanto los impactos que recibió el recurso forestal como su recuperación potencial (Pinazo et al. 2003; Gasparri et al. 2003).

En particular, la Región Chaqueña presenta una tasa de deforestación elevada, lo que la convierte en una de las más activas áreas de pérdida de bosque (Hansen et al. 2013; Baumann et al. 2016). Además, los bosques de la región, como en otros lados del mundo, son sometidos a diferentes usos, incluyendo las cortas selectivas sobre especies valiosas, que pueden promover su degradación. Trabajos previos muestran que los procesos de degradación pueden afectar grandes extensiones de bosques secos, pero generalmente subestimados por falta de estimaciones frente a la deforestación (le Polain de Woaroux and Lambin 2012). El palo santo, Bulnesia sarmientoi Lorentz ex Griseb. (Zygophyllaceae), es una especie endémica en Sudamérica. Se distribuye en el Chaco Seco americano, incluyendo el norte de la Argentina, el este de Bolivia y el oeste de Paraguay. Ocasionalmente, forma rodales casi puros denominados palosantales o puede formar parte de las especies arbóreas del bosque chaqueño típico (Cabrera 1976). El palo santo es una de las especies con maderas duras típicas de la región chaqueña (Giménez and Moglia 2003). En los últimos 10 años aumentó la demanda internacional de la madera de palo santo, por lo cual hubo un aumento en la exportación de rollos y postes desde la Argentina hacia países de extremo oriente (CITES 2010). Su principal uso en nuestro país es el artesanal (e.g., tornería, tallados, pisos, postes) y también se lo emplea en construcciones debido a que su madera es muy durable. En la Argentina, en el período 2008-2010, la especie fue recategorizada en términos de amenaza para su conservación, y pasó del apéndice III al II en la Convención sobre el Comercio Internacional de Especies Amenazadas de Fauna y Flora Silvestre (CITES). La CITES establece que la exportación de cualquier espécimen incluido dentro del apéndice II requiere de una previa concesión y permiso de exportación que se obtiene sólo al demostrar que no se está afectando la supervivencia de la especie (Ley 22344 $\mathrm{MJyDH}$ ). Además dela demandainternacional, existe un uso doméstico tradicional orientado a la producción de postes y carpintería rural (i.e., varillas, artesanías y herramientas) que suele ocurrir de manera informal. Este tipo de aprovechamiento esta poco documentado y se desconocen los impactos potenciales que puede generar.

Hasta el momento, son escasos los estudios que permiten establecer referencias de la estructura de bosques con $B$. sarmientoi bien desarrollados y evaluar los impactos de los aprovechamientos y la posterior evolución de la población de esta especie. En la provincia de Formosa, Waller (2009) analizó dos sitios (con y sin aprovechamiento) con B. sarmientoi y sugiere que se compromete el potencial productivo de la especie. Por otra parte, Mereles and Pérez de Molas (2008) realizaron un censo en Paraguay en dos poblaciones de palo santo y encontraron una distribución irregular en el número de árboles según tipos de formación boscosa (palosantal y bosque chaqueño), por lo que recomiendan realizar estudios que contemplen la dinámica de regeneración y el crecimiento anual. Por su lado, Giménez et al. (2007) estudiaron el crecimiento de la especie a partir del análisis de fustes de individuos provenientes de Formosa.

El objetivo del presente trabajo fue caracterizar estructuras forestales de bosques del Chaco Seco con presencia de B. sarmientoi y luego, a partir de datos de re-mediciones de parcelas permanentes, calcular tasas de crecimiento de $B$. sarmientoi para individuos de distintos tamaños. Finalmente, tomando como referencia la estructura de parcelas forestales permanentes que fueron intervenidas entre las re-mediciones, se evaluó el tiempo de recuperación de la población de $B$. sarmientoi por medio del método de proyección de tabla de rodal. 


\section{Materiales y Métodos}

\section{Área de estudio}

$\mathrm{El}$ área de estudio $\left(24^{\circ} 49^{\prime} 15^{\prime \prime}-22^{\circ} 01^{\prime} 36^{\prime \prime} \mathrm{S}\right.$; $62^{\circ} 51^{\prime} 58^{\prime \prime}-61^{\circ} 02^{\prime} 06^{\prime \prime}$ O) está ubicada en el sector norte del Chaco Seco de la Argentina. Involucra el este de la provincia de Salta (departamentos de Rivadavia, Anta y San Martín) y el oeste de la provincia de Formosa (departamentos de Bermejo y Patiño). La ecorregión del Chaco Seco es una región extensa de bosques xerófitos, y es la segunda región más grande después del Amazonas (Bonino 2006). Las especies del dosel son Aspidosperma quebracho-blanco, Schinopsis lorentzii, Prosopis nigra, Prosopis alba y Ziziphus mistol. Generalmente, debajo del dosel se encuentran Bouganvillea praecox, Capparis spp., Salta triflora, Celtis tala, Acacia spp., entre otras (Cabrera 1976; Burkart 1999). En la Argentina, B. sarmientoi se distribuye hacia el norte del Chaco Seco, y se encuentra sólo en las provincias de Salta, Formosa y Chaco. Esta área tiene un interés particular debido a la concentración de aserraderos forestales (Figura 1).

\section{Diseño de muestreo}

En el 2007 se establecieron 21 conglomerados forestales permanentes dentro del área de estudio. Cada conglomerado estuvo compuesto por cuatro juegos de parcelas concéntricas ubicadas en los vértices de un cuadrado de 100 m de lado; el centro de las mismas se registró con GPS. Cada juego de parcelas concéntricas estuvo compuesto por una parcela de $1000 \mathrm{~m}^{2}$ para los árboles con diámetro a la altura del pecho (DAP) $>20 \mathrm{~cm}$ y por una segunda parcela de $500 \mathrm{~m}^{2}$ para los árboles menores, con DAP $>10 \mathrm{~cm}$. Para cada árbol se determinó la especie y se midió el DAP con cinta diamétrica. Además, los árboles con DAP $>20 \mathrm{~cm}$ fueron identificados con chapa numerada y se registró la ubicación mediante rumbo y distancia al centro de la parcela para permitir su re-medición. Estos conglomerados se ubicaron en distintos tipos de uso y propiedad de la tierra, incluyendo tierras comunitarias aborígenes, áreas protegidas (RN Pizarro y RN Formosa), propiedades fiscales y tierras fiscales, y parte de ellos fueron remedidos en el año 2012. El método de medición fue descripto y utilizado
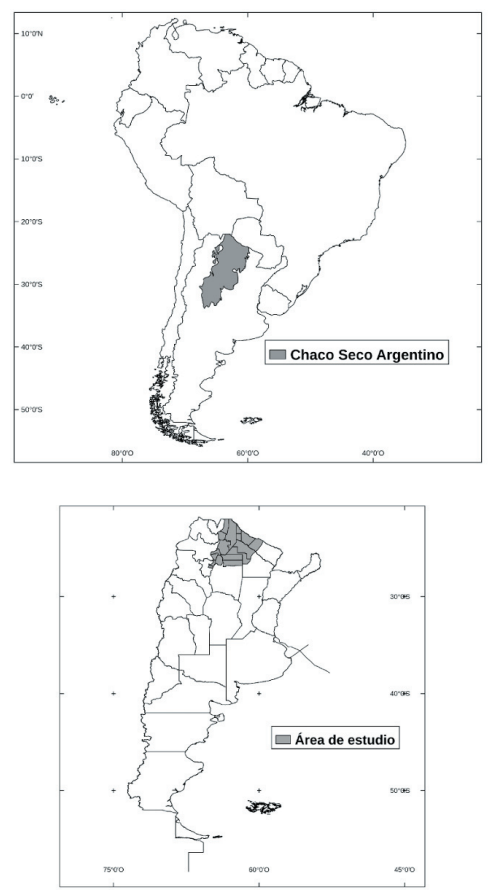

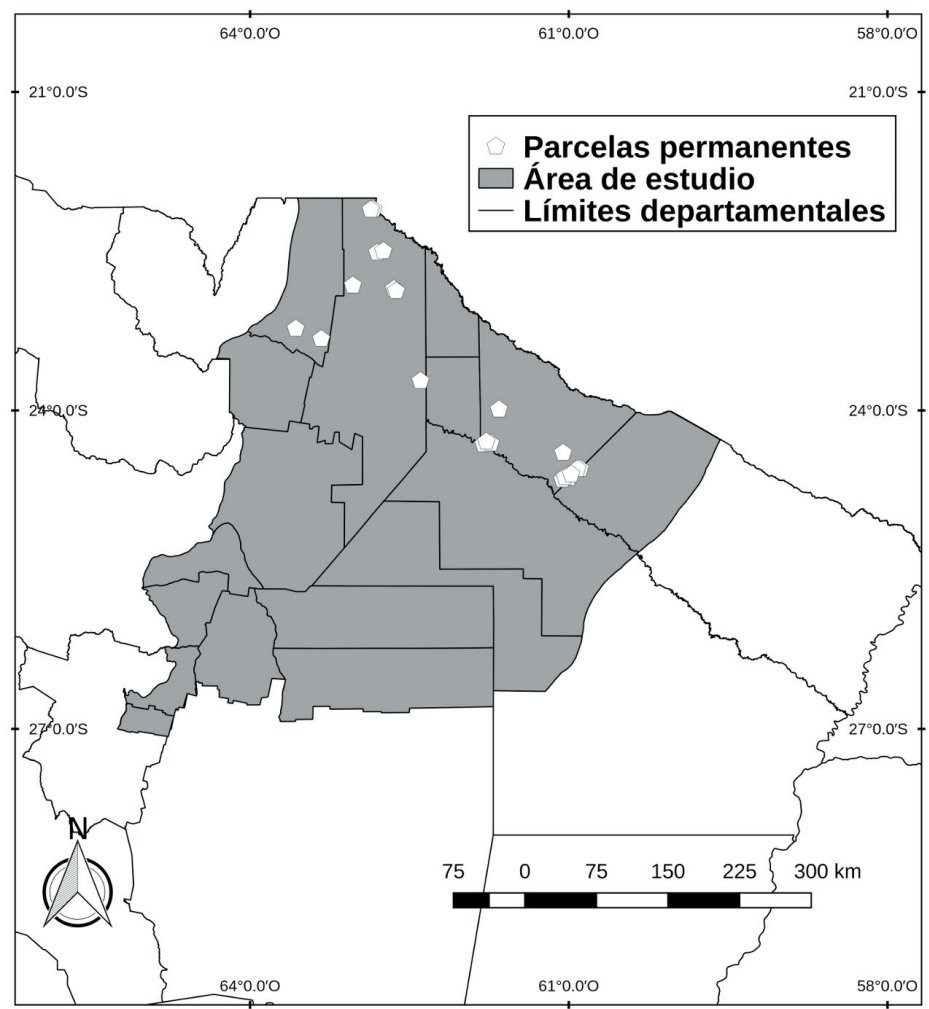

Figura 1. Área de estudio y ubicación de las parcelas permanentes establecidas en 2007.

Figure 1. Study area and distribution of permanent plots at 2007. 
para estimar biomasa de bosque del Chaco Seco en Gasparri and Baldi (2013).

\section{Parámetros estructurales}

En base a los datos relevados en las parcelas, para cada conglomerado se calculó el área basal (AB), la densidad (B) y la frecuencia (F) de la especie en función de su presencia en las 4 parcelas del conglomerado (i.e., frecuencia de $0,25,50,75$ ó $100 \%$ ). Se calcularon los porcentajes relativos de estos tres parámetros para construir el índice de valor de importancia (IVI). Este índice resulta de la sumatoria de los valores relativos $A B, D$ y $F$ para cada especie. Valores altos y bajos de IVI indican mayor o menor importancia estructural, respectivamente (Lamprecht 1990; Pinazo et al. 2003). Con los datos de re-medición de los individuos de B. sarmientoi que tuvieron DAP> $20 \mathrm{~cm}$ se calculó el incremento periódico anual (IPA) de B. sarmientoi para distintos rangos de tamaño.

\section{Análisis de datos}

Se realizó un análisis de agrupamiento de las parcelas que tuvieron presencia de B. sarmientoi. Se utilizó el IVI como valor calificativo de parcelas para determinar diferentes rodales. Se empleó el método de Ward como técnica aglomerativa. Se obtuvieron las probabilidades de los grupos resultantes por método bootstraping, simulando 1000 re-muestreos (Suzuki and Shimodaira 2006; R Core Team 2017).

\section{Proyección de tablas de rodal}

Para explorar el impacto de los aprovechamientos sobre la población de palo santo a nivel de rodal se realizaron simulaciones de cortas y de la posterior evolución de la estructura diamétrica de la especie mediante la técnica de simulación de tabla de rodal. Para poder calcular las transiciones de una clase de tamaño a otra se calculó el incremento periódico anual (IPA) por clase diamétrica en base a las mediciones repetidas de los árboles de palo santo en el año 2007 y 2012. Tomando como referencia la estructura de uno de los conglomerados sometido a cortas entre la medición del 2002 y la del 2007, se evaluaron dos tipos de intervención alternativas sobre la estructura inicial (2007), en función de reglamentaciones aplicadas sobre el palo santo. La primera consiste en conservar $20 \%$ de los individuos en todas las clases de tamaño para regeneración del recurso, mientras que la segunda regla corresponde a la aplicación de la regla de diámetro mínimo de corta (DMC), por lo cual se retienen en el rodal los individuos con DAP menor a $40 \mathrm{~cm}$ (Waller 2009). Luego, con los valores de IPA obtenidos se proyectó a 50 años el crecimiento de los árboles remanentes (Oliver and Larson 1996; Husch et al. 2002) y la distribución de tamaños de la especie. De esta manera, se contrasta la evolución de la población de palo santo remanente luego del aprovechamiento observado en el conglomerado (medición 2012) con las intervenciones simuladas. Los supuestos para esta proyección de rodal de $B$. sarmientoi fueron mantener constante la clase inferior (incorporación constante en la clase de $10 \mathrm{~cm}$ DAP), la no competencia interespecífica (es decir, no hay competencia entre especies por el espacio) y la homogeneidad de amplitud por clase diamétrica (i.e., un número constante de árboles en cada clase diamétrica a lo largo del tiempo).

\section{Resultados}

El análisis de agrupamiento de los 21 conglomerados en base al valor IVI de cada especie arroja 3 grupos identificados (Figura 2). En una primera separación, los conglomerados C16, C17 y C21 forman el primer grupo (A), que se corresponde con las formaciones naturales de palosantales en bañados o de suelos inundables, con los valores más altos de IVI. El segundo grupo (B) estuvo formado por los conglomerados C24 y C26, con dominancia compartida en Tabebuia nodosa (palo cruz) y B. sarmientoi. El último grupo (C) estuvo formado por el resto de los conglomerados y representó para $B$. sarmientoi los menores valores de IVI, ya que son estructuras dominadas por Aspidosperma quebracho-blanco y Schinopsis lorentzii (Tabla $1)$.

La extrapolación del número de individuos por hectárea (individuos/ha) de B. sarmientoi discriminados por clases diamétricas $(\mathrm{cm})$ arrojó que el primer grupo (A) presentó una distribución diamétrica con preponderancia de individuos en las clases inferiores y un total de 286 individuos/ha. Los grupos B y C presentaron una distribución diamétrica más regular (38 individuos/ha y 25 individuos/ ha, respectivamente), sin mostrar diferencias significativas entre ellos. Las restantes especies forestales acompañantes presentaron una distribución diamétrica del tipo J invertida. En el primer grupo (A), las especies acompañantes no superaron los 113 individuos/ha; el segundo grupo (B) presentó 276 individuos / 
Tabla 1. Índice de valor de importancia (IVI), área basal (AB), densidad (D) y frecuencia (F) en valores relativos (\%) para tres grupos resultantes del agrupamiento de veintiún conglomerados con presencia de $B$. sarmientoi. Table 1. Importance value index (IVI), basal area (AB), density (D) and frequency (F) in percentage $(\%)$ for three groups resulting from cluster analysis for twenty-one plots with $B$. sarmientoi presence.

\begin{tabular}{|c|c|c|c|c|c|c|c|c|c|c|c|c|}
\hline \multirow[t]{2}{*}{ Especies } & \multicolumn{4}{|c|}{$\begin{array}{c}\text { Grupo A } \\
n=3\end{array}$} & \multicolumn{4}{|c|}{$\begin{array}{c}\text { Grupo B } \\
n=2\end{array}$} & \multicolumn{4}{|c|}{$\begin{array}{c}\text { Grupo } C \\
n=16\end{array}$} \\
\hline & $\mathrm{AB}$ & $\mathrm{D}$ & $\mathrm{F}$ & IVI & $\mathrm{AB}$ & $\mathrm{D}$ & $\mathrm{F}$ & IVI & $\mathrm{AB}$ & $\mathrm{D}$ & $\mathrm{F}$ & IVI \\
\hline Acacia caven & & & & & & & & & 0.5 & 0.6 & 0.2 & 1.2 \\
\hline Acanthosyris falcata & 0.3 & 0.5 & 2.0 & 2.8 & & & & & 2.0 & 2.3 & 1.0 & 5.2 \\
\hline Acacia gilliesii & & & & & & & & & 0.6 & 0.7 & 1.0 & 2.2 \\
\hline Acacia praecox & 2.2 & 3.2 & 2.4 & 7.7 & 0.5 & 0.7 & 2.3 & 3.5 & 4.1 & 5.6 & 5.0 & 14.7 \\
\hline Acacia sp. & 1.2 & 1.6 & 2.0 & 4.8 & & & & & & & & \\
\hline Aspidosperma quebracho-blanco & 8.7 & 6.9 & 19.3 & 35.0 & 1.1 & 1.3 & 5.6 & 7.9 & 16.2 & 14.5 & 11.1 & 41.7 \\
\hline Bougainvillea praecox & 2.3 & 2.7 & 9.8 & 14.8 & 1.4 & 1.4 & 2.3 & 5.1 & 4.1 & 4.8 & 6.3 & 15.2 \\
\hline Bulnesia sarmientoi & 73.3 & 71.5 & 25.2 & 170.1 & 13.7 & 10.7 & 12.4 & 36.9 & 8.4 & 7.0 & 8.2 & 23.6 \\
\hline Castela coccinea & & & & & & & & & 0.2 & 0.3 & 0.7 & 1.2 \\
\hline Calycophyllum multiflorum & & & & & & & & & 1.7 & 1.4 & 1.3 & 4.4 \\
\hline Caesalpinia paraguariensis & & & & & 2.3 & 1.4 & 3.3 & 7.0 & 3.4 & 3.1 & 3.4 & 9.9 \\
\hline Capparis retusa & & & & & & & & & 0.7 & 1.0 & 1.4 & 3.1 \\
\hline Capparis salicifolia & & & & & & & & & 1.5 & 2.1 & 3.4 & 7.0 \\
\hline Capparis speciosa & & & & & 0.6 & 0.7 & 2.3 & 3.5 & 1.5 & 2.2 & 3.9 & 7.6 \\
\hline Capparis tweediana & & & & & & & & & 0.0 & 0.1 & 0.2 & 0.3 \\
\hline Ceiba insignis & 0.4 & 0.5 & 2.0 & 2.9 & 0.7 & 0.3 & 2.3 & 3.3 & 0.0 & 0.0 & 0.2 & 0.3 \\
\hline Cercidium praecox & 1.2 & 1.2 & 3.9 & 6.3 & & & & & 1.2 & 1.5 & 1.7 & 4.5 \\
\hline Celtis tala & & & & & & & & & 0.4 & 0.5 & 0.7 & 1.6 \\
\hline Geoffroea decorticans & & & & & & & & & 0.2 & 0.1 & 0.4 & 0.7 \\
\hline Mimozygathus carinatus & 0.9 & 1.1 & 3.9 & 5.9 & 0.5 & 0.7 & 2.3 & 3.5 & 0.5 & 0.6 & 1.0 & 2.0 \\
\hline Patagonula americana & & & & & & & & & 0.3 & 0.4 & 0.7 & 1.3 \\
\hline Phyllostylon rhamnoides & & & & & & & & & 0.1 & 0.0 & 0.2 & 0.3 \\
\hline Pizonia zapallo & & & & & & & & & 0.4 & 0.2 & 0.4 & 1.0 \\
\hline Prosopis alba & & & & & 2.2 & 1.9 & 6.7 & 10.8 & 3.3 & 2.5 & 2.2 & 7.9 \\
\hline Prosopis kuntzei & & & & & & & & & 3.4 & 2.7 & 3.8 & 9.9 \\
\hline Prosopis nigra & & & & & 6.6 & 5.1 & 6.7 & 18.3 & 2.3 & 1.9 & 2.5 & 6.8 \\
\hline Prosopis rucsifolia & & & & & & & & & 0.4 & 0.5 & 0.7 & 1.6 \\
\hline Ruprechtia triflora & 4.1 & 6.3 & 10.6 & 21.1 & 9.5 & 13.0 & 15.8 & 38.3 & 13.8 & 18.2 & 9.5 & 41.6 \\
\hline Sapium haematospermum & 0.5 & 0.2 & 2.4 & 3.1 & & & & & & & & \\
\hline Schinopsis lorentzii & & & & & 2.0 & 1.4 & 6.7 & 10.0 & 5.7 & 3.6 & 6.5 & 15.8 \\
\hline Sideroxylon obtusifolium & & & & & 1.2 & 1.4 & 2.3 & 4.8 & 2.4 & 2.0 & 3.5 & 7.9 \\
\hline Tabebuia nodosa & 2.2 & 1.3 & 8.3 & 11.7 & 55.4 & 57.9 & 22.4 & 135.8 & 5.9 & 5.3 & 4.9 & 16.1 \\
\hline Ximenia americana & & & & & & & & & 0.6 & 0.6 & 1.2 & 2.3 \\
\hline Ziziphus mistol & 2.7 & 2.9 & 8.3 & 13.9 & 0.8 & 0.3 & 2.3 & 3.4 & 12.7 & 11.9 & 9.7 & 34.3 \\
\hline Otras especies & & & & & 1.6 & 1.7 & 4.5 & 7.9 & 1.9 & 1.7 & 3.1 & 6.8 \\
\hline Total & 100 & 100 & 100 & 300 & 100 & 100 & 100 & 300 & 100 & 100 & 100 & 300 \\
\hline
\end{tabular}

ha, mientras que el tercer grupo (C) superó los 330 individuos/ha. La abundante presencia de T. nodosa (177 individuos/ha) como especie acompañante en el grupo B fue la principal diferencia con el grupo C (Figura 3).

Durante el período 2007-2012, de las parcelas permanentes se obtuvo un incremento periódico anual (IPA) de $1.14 \mathrm{~mm} /$ año para un total de 126 ejemplares de Bulnesia sarmientoi (Figura 4). Para la simulación se eligió el conglomerado nro. 21 (Sta. Victoria Este, Salta), ya que este último tuvo cortas y aprovechamientos exclusivos de palo santo, lo cual refleja una situación frecuente en el área de estudio. La simulación de un aprovechamiento del $80 \%$ arrojó un menor número de árboles respecto de la simulación con DMC (Tabla 2). Al final del período proyectado, la simulación del $80 \%$ aprovechado no superó los 10 individuos/ha de palo santo, mientras que al final de simular la regla de diámetro mínimo de corta, alcanzó los 40 individuos/ha. La regla de aprovechar el $80 \%$ de palo santo nunca superó el aprovechamiento ocurrido en 2012 en el conglomerado nro. 21 (Figura 5). 


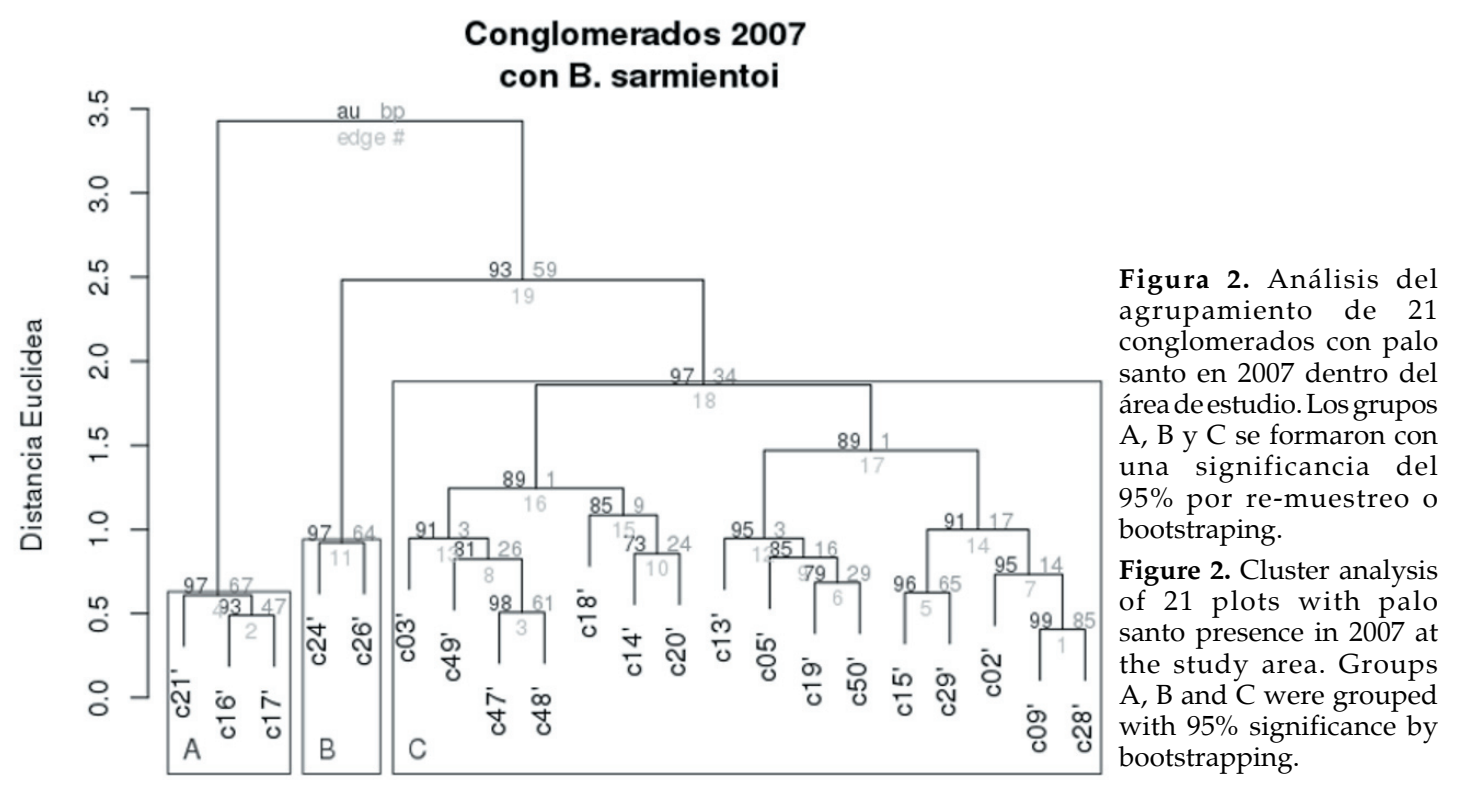

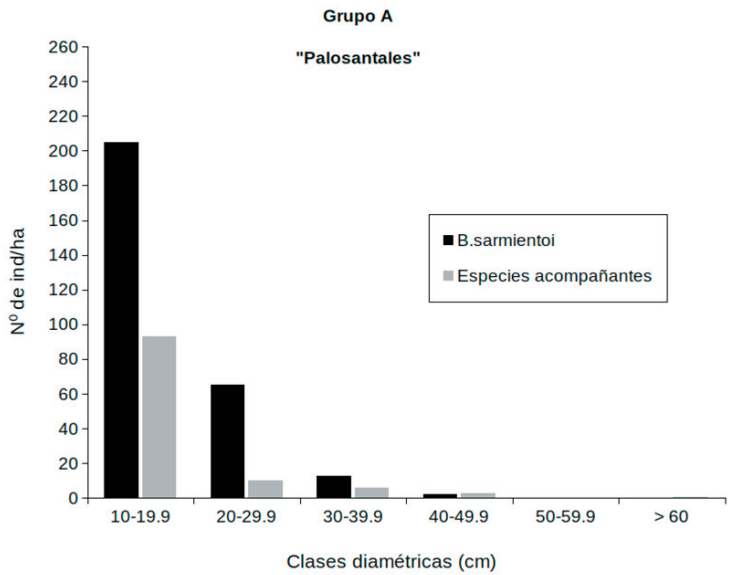

Grupo C

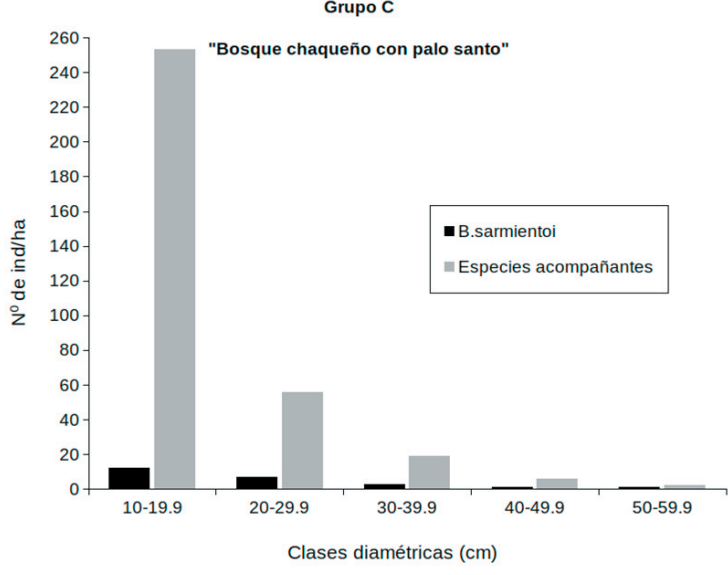

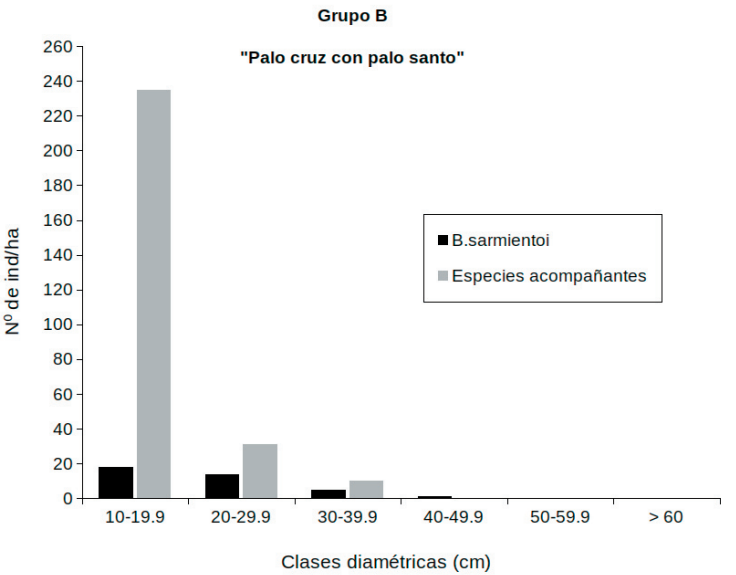

Figura 3. Distribución de clases diamétricas para $B$. sarmientoi y especies acompañantes. El grupo A tuvo la mayor cantidad de individuos por hectárea de palo santo. Los grupos $\mathrm{B}$ y $\mathrm{C}$ concentran más individuos de $T$. nodosa, A. quebracho-blanco, S. lorentzii y Z. mistol respecto del palo santo.

Figure 3. Diameter class distribution of B. sarmientoi and accompanying species. Group $\mathrm{A}$ has the greatest number of palo santo individuals per hectare. Groups B and C host more individuals of T. nodosa, A. quebracho-blanco, $S$. lorentzii and Z. mistol with regard to palo santo. 


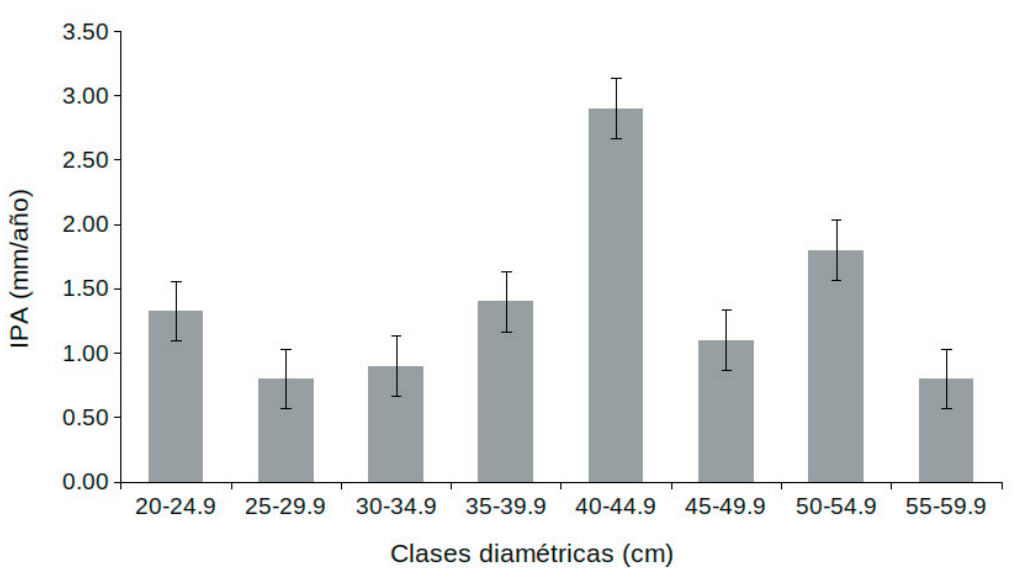

Figura 4. Incremento periódico anual (IPA) para las diferentes clases diamétricas de $B$. sarmientoi. Las líneas verticales indican error estándar. El crecimiento promedio anual general encontrado fue 1.14 $\mathrm{mm}(\mathrm{n}=126)$.

Figure 4. Periodic annual increment (IPA) for the different diametric class of $B$. sarmientoi. Vertical lines represent standard error. General mean annual increment was $1.14 \mathrm{~mm}$ $(n=126)$.

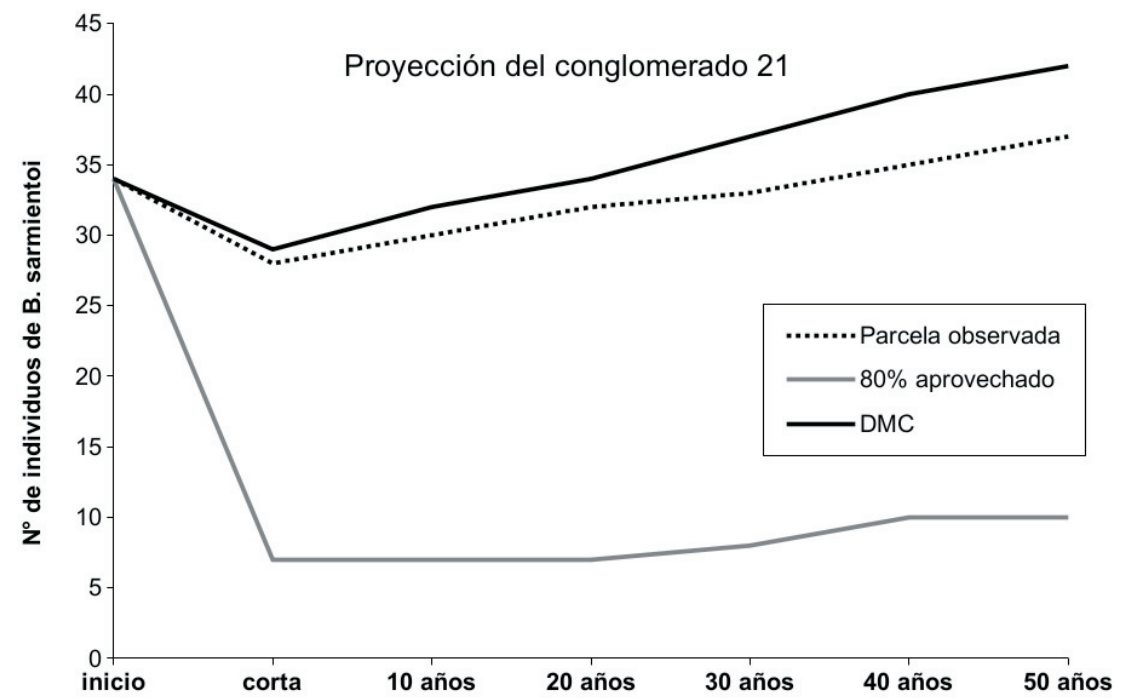

Figura 5. Proyección de rodal de B. sarmientoi en el conglomerado $\mathrm{n}^{\circ} 21$ con cortas observadas en terreno durante 2012 (línea punteada). Al final de la simulación se observa un mayor número de individuos de palo santo con diámetro mínimo de corta (DMC), con respecto a la simulación de un aprovechamiento que conserva sólo $20 \%$ de los ejemplares de palo santo $(80 \%)$.

Figure 5. Stand projection of B. sarmientoi in plot $n^{\circ} 21$ with logging observations at terrain in 2012 (dotted line). Minimum cut diameter simulation (DMC) had the greatest number of palo santo individuals, in relation to $80 \%$ logging simulation over 50 years.

Tabla 2. Proyección de tablas de rodal de B. sarmientoi a 50 años. Clases diamétricas en cm (CD). Incremento periódico para 10 años en $\mathrm{cm}$ (IP) de cada clase diamétrica. Tasa de paso o rotación de rodal (TP). Individuos por hectárea medidos en 2007 y 2012 (ind/ha). La primera simulación conserva solo $20 \%$ de los individuos ("80\%"). La segunda simulación aprovecha árboles con un diámetro mínimo de corta de $40 \mathrm{~cm}$ ("DMC"). Table 2. Stand projection tables of B. sarmientoi at 50 years. Diametric class in $\mathrm{cm}$ (CD). Periodic increment of 10 years in $\mathrm{cm}(\mathrm{IP})$ each class. Stand rotation rate (TP). Individuals per hectare measured in 2007 and 2012 (ind/ha). First simulation conserves only $20 \%$ of individuals (" $80 \%$ "). Second logging simulation cuts trees with a minimum diameter of $40 \mathrm{~cm}$ ("DMC").

\begin{tabular}{cccccccccccccccccc}
\hline CD & IP & TP & \multicolumn{3}{c}{$\begin{array}{c}\text { ind/ha ind/ha } \\
2007\end{array}$} & $\begin{array}{c}\text { ind/ha } \\
\text { "80\%" }\end{array}$ & $\begin{array}{c}10 \\
\text { años }\end{array}$ & $\begin{array}{c}20 \\
\text { años }\end{array}$ & $\begin{array}{c}30 \\
\text { años }\end{array}$ & $\begin{array}{c}40 \\
\text { años }\end{array}$ & $\begin{array}{c}50 \\
\text { años }\end{array}$ & $\begin{array}{c}\text { ind/ha } \\
\text { "DM" años }\end{array}$ & $\begin{array}{c}20 \\
\text { años }\end{array}$ & $\begin{array}{c}30 \\
\text { años años }\end{array}$ & $\begin{array}{c}50 \\
\text { años }\end{array}$ \\
\hline $20-25$ & 1.3 & 0.27 & 10 & 7 & 2 & 2 & 2 & 2 & 2 & 2 & 10 & 10 & 10 & 10 & 10 & 10 \\
$25-30$ & 0.8 & 0.16 & 11 & 11 & 2 & 2 & 2 & 3 & 3 & 3 & 11 & 12 & 13 & 13 & 14 & 14 \\
$30-35$ & 0.9 & 0.18 & 8 & 5 & 2 & 2 & 2 & 2 & 2 & 2 & 8 & 8 & 9 & 9 & 10 & 10 \\
$35-40$ & 1.4 & 0.28 & 3 & 3 & 1 & 1 & 1 & 1 & 1 & 1 & 0 & 1 & 3 & 3 & 4 & 5 \\
$40-45$ & 2.9 & 0.58 & 1 & 1 & 0 & 0 & 0 & 0 & 1 & 1 & 0 & 0 & 0 & 1 & 1 & 2 \\
$45-50$ & 1.1 & 0.22 & 1 & 0 & 0 & 0 & 0 & 0 & 1 & 1 & 0 & 0 & 0 & 0 & 1 & 1 \\
$50-55$ & 1.8 & 0.36 & 0 & 1 & 0 & 0 & 0 & 0 & 0 & 0 & 0 & 0 & 0 & 0 & 0 & 0 \\
$55-60$ & 0.8 & 0.16 & 0 & 0 & 0 & 0 & 0 & 0 & 0 & 0 & 0 & 0 & 0 & 0 & 0 & 0 \\
Total & & & 34 & 28 & 7 & 7 & 7 & 8 & 10 & 10 & 29 & 32 & 34 & 37 & 40 & 42 \\
\hline
\end{tabular}




\section{Discusión}

El enfoque clásico de análisis de estructura y dinámica de rodal empleado en el presente trabajo resultó clave para idealizar cuánto y de qué manera existe $B$. sarmientoi en el norte del Chaco Seco (Oliver and Larson 1996). La aproximación del concepto de estructura permitió diferenciar formaciones forestales de B. sarmientoi observables en el área de estudio y que pueden servir para desarrollar nuevas estrategias de manejo y conservación en la escala de paisaje. Sin embargo, algunos de los grupos A y B estuvieron formados por unos pocos conglomerados ( 3 y 2 , respectivamente), a diferencia del grupo C, que estuvo formado por 16 conglomerados. Esto sugiere que los palosantales y las asociaciones con T. nodosa (palo cruz) son rodales circunscriptos en áreas puntuales donde el suelo se inunda frecuentemente; no así los bosques chaqueños típicos con presencia de B. sarmientoi (Grupo C), donde los suelos no se inundan con frecuencia. Estos rodales de suelo profundo con dominancia de quebrachos y presencia variable de palo santo constituyen los rodales más comunes dentro del área de estudio. Estos datos concuerdan con las observaciones florísticas de esta especie, sus descripciones biogeográficas y evaluaciones ambientales según Cabrera (1976), Giménez and Moglia (2003), Mereles and Pérez de Molas (2008) y Cop 15 (2010).

En nuestros análisis determinamos un IPA de $1.14 \mathrm{~mm}$ para Bulnesia sarmientoi como resultado de una re-medición de parcelas permanentes. Giménez et al. (2008) determinaron, mediante técnicas dendrológicas, un incremento medio anual (IMA) de $4.18 \mathrm{~mm}$ para una serie temporal de 104 años, para lo cual demarcaron anillos sobre rodajas de troncos de $5 \mathrm{~cm}$ de espesor $(n=10)$ en leños de diámetro $>30 \mathrm{~cm}$. No obstante, estos dos valores de incremento en diámetro no llegan a los valores de referencia (5 mm/año) para que se la considere como de rápido crecimiento en la Región Chaqueña (Giménez et al. 2007). Se encontraron distintos valores de crecimiento para las diferentes clases diamétricas (Figura 4), lo que representa una mejora en relación a la aplicación de un único valor para la especie. Sin embargo, el seguimiento de mediciones en las parcelas permanentes y la ampliación del número de árboles medidos a lo largo del tiempo son necesarios para reducir la incertidumbre sobre los datos de crecimiento de B. sarmientoi (Gasparri et al. 2003; Araujo et al. 2007).
Las simulaciones exploradas en este trabajo permitieron diferenciar dos reglas silvícolas aplicadas a $B$. sarmientoi y contrastar su evolución posterior contra la estructura remanente de una corta real registrada entre las re-mediciones. Usar DMC mantiene mayor cantidad de árboles en las clases de pequeño tamaño a lo largo de 50 años. Al aplicar DMC en formaciones de palo santo se limita a extraer únicamente trozas de grandes volúmenes, pero es importante señalar que por sí sola, esta regla no garantiza la regeneración ni la sustentabilidad del recurso (Gasparri et al. 2003). Por otro lado, la corta del $80 \%$ de ejemplares en cada clase de tamaño de palo santo genera un menor recambio de individuos y pone en duda la mínima recuperación del recurso en 50 años. Esta situación sugiere que esta intervención sería muy severa y que afectaría principalmente a las clases diamétricas inferiores $(<20 \mathrm{~cm}$ de DAP). La corta concentradas en tamaños pequeños es una situación común vinculada a los aprovechamientos de postes y varillas para alambrados y actividades rurales. Este tipo de cortas puede ser difícil de controlar, ya que muchas veces son productos que no entran en circuitos comerciales formales y, desde ya, al no estar vinculado con la exportación no se le aplican los requerimientos de mercado asociado a CITES. La elección final de una u otra regla silvícola no fue aquí analizada. Sin embargo, un análisis de simulación con ambas reglas silvícolas sobre las restantes estructuras forestales encontradas en este trabajo de B. sarmientoi (i.e., palosantales, palo cruz con palo santo y bosque chaqueño con palo santo) podría arrojar otros escenarios de oportunidades para aprovechar el recurso.

A raíz del ordenamiento territorial en el país (Piquer-Rodríguez et al. 2015) y las normativas del apéndice II para el comercio internacional de palo santo (CITES), sólo se aprobarán extracciones de madera de $B$. sarmiento $i$ provenientes de planes de manejo sustentables a partir de 2013 (Waller 2009; SAyDS 2013). Sin embargo, la actividad de extracción de palo santo en estas provincias durante 2011-2013 no disminuyó. Dentro de la situación actual de amenaza de $B$. sarmientoi, la extracción de rollos, fustes, postes, etc. continúa ocurriendo, en muchos casos, sin planes de manejo (i.e., extracción en comunidades aborígenes).

Actualmente en la Argentina, muchas áreas que incluyen la distribución del palo santo tienen la posibilidad de ser convertidas en pasturas o para usos agrícolas. Y donde no 
existe un protocolo unificado dentro de las provincias que comparten su distribución (Salta, Formosa y Chaco), la continuidad de la especie como recurso maderero de la región podría verse comprometida. Para evitar esta situación, sería necesario avanzar en la gestión planificada del recurso, ejerciendo controles rigurosos en el terreno y fomentando la elaboración y el aumento del valor agregado de los productos obtenidos. Para ello es necesario disponer de mucha información sobre la ecología y el manejo de la especie, como la provista por el Inventario Forestal Nacional de Palo Santo (SAyDS 2013), que representa un avance significativo para $B$. sarmientoi. Sin embargo, la información sobre aspectos como la regeneración y el crecimiento de la especie resultan prioritarios. En ese sentido, este trabajo resulta un primer avance al presentar datos de estructura de bosque que sirvan de referencia, y al proporcionar información de crecimiento periódico (IPA) para diferentes tamaños de árbol, lo cual permitiría su aplicación sobre datos de inventario forestal y sin necesidad de saber la edad de los individuos.

Agradecimientos. Los autores agradecen al financiamiento del proyecto FONCyT - PICTO OTNA 2011 N 97 y el fondo para la conservación de la naturaleza del Banco Galicia Argentina (FOCA) para la realización de este trabajo. A la Administración de Parques Nacionales (RN Pizarro y RN Formosa). Comunidades aborígenes Wichí de Salta y Formosa. Al ingeniero Pedro Fernández y Dra. Carla Rueda por sus valiosos comentarios que dieron forma al presente trabajo.

\section{REFERENCIAS}

Araujo, P., M. Juárez de Galíndez, and M. Iturre. 2007. Crecimiento de las especies principales de un bosque en regeneración del Chaco Santiagueño. Quebracho 14:36-46.

Baumann, M., I. Gasparri, M. Piquer-Rodríguez, G. Gavier Pizarro, P. Griffiths, P. Hoster, and T. Kuemmerle. 2016. Carbon emission from agricultural expansion and intensification in the Chaco. Global Change Biology 23(5):19021916. DOI: $10.1111 /$ gcb.13521.

Bonino, E. 2006. Changes in carbon pools associated with a land-use gradient in the Dry Chaco, Argentina. Forest Ecology and Management 223:183-189.

Burkart, R., N. O. Bárbaro, R. O. Sánchez, and D. A. Gómez. 1999. Eco-regiones de la Argentina. Secretaría de Recursos Naturales y Desarrollo Sustentable. Administración de Parques Nacionales.

Cabrera, A. 1976. Regiones fitogeográficas argentinas. Segunda Edición. Enciclopedia Argentina de Agricultura y Jardinería. Editorial ACME. Buenos Aires. Pp. 1-85.

Convención sobre el Comercio Internacional de Especies Amenazadas de Fauna y Flora silvestres. 2010. Decimoquinta reunión de conferencia de las partes (Cop 15). Examen de las propuestas de enmienda a los apéndices I y II. Doha, Qatar. URL: www.cites.org.

Convención sobre el Comercio Internacional de Especies Amenazadas de Fauna y Flora silvestres. 2012. Vigésima reunión del comité de flora (CoP 20). Bulnesia sarmientoi. Informe Argentina. Dublin, Irlanda. URL: www.cites.org.

Edwards, D. P., J. A. Tobias, D. Sheil, E. Meijaard, and W. F. Laurance. 2014. Maintaining ecosystem function and services in logged tropical forests. Trends in Ecology and evolution 29:511-520. DOI: 10.1016/j.tree.2014.07.003.

Gasparri, N. I., and G. Baldi. 2013. Regional patterns and controls of biomass in semiarid woodlands: lessons from the Northern Argentina Dry Chaco. Regional Environmental Change. 13(6):1131-1144. DOI:10.1007/s10113-013-0422$\mathrm{x}$.

Gasparri, N., M. Pinazo, and J. Goya. 2003. Crecimiento individual y diámetro mínimo de corta de Juglans australis: simulación de intervención en un rodal maduro en el noroeste de argentina. Yvyrareta 11:27-33.

Giménez, A. M., and J. G. Moglia. 2003. Árboles del Chaco Argentino. Guía para el reconocimiento dendrocronológico. Facultad de Ciencias Forestales. Secretaría de Ambiente y Desarrollo Sustentable. Pp. 307.

Giménez, A. M., P. Hernández, R. Geréz, and C. Spagarino. 2007. Anatomía de leño y crecimiento de Palo Santo (Bulnesia sarmientoi Lorentz ex Griseb. Zygophyllaceae). Quebracho 14:23-35.

Giménez, A. M., P. Hernández, C. Spagarino, J. G. Moglia, and N. A. Ríos. 2008. Calidad de madera y potencialidad de crecimiento de palo santo (Bulnesia sarmientoi Lorentz ex Griseb.) Yvyrareta 15:60-67.

Hansen, M. C., P. V. Potapov, R. Moore, M. Hancher, S. A. Turubanova, A. Tyukavina, D. Thau, S. V. Stehman, S. J. Goetz, T. R. Loveland, A. Kommareddy, A. Egorov, L. Chini, C. O. Justice, and J. R. G. Townshend. 2013. Highresolution global maps of 21st-century forest cover change. Science 342:850-3. DOI:10.1126/science.1244693.

Husch, B., T. W. Beers, and J. A. Kershaw Jr. 2002. Forest mensuration. John Wiley \& Sons.

Lamprecht, H. 1990. Silvicultura en los trópicos: los ecosistemas forestales en los bosques tropicales y sus especies arbóreas; posibilidades y métodos para un aprovechamiento sostenido. GTZ. Rossdorf, Alemania. Pp. 155.

le Polain de Waroux, Y., and E. F. Lambin. 2012. Monitoring degradation in arid and semi-arid forests and woodlands: The case of the argan woodlands (Morocco). Appl. Geogr. 32:777-786. DOI:10.1016/j.apgeog.2011.08.005.

Mereles, F., and L. Pérez de Molas. 2008. Bulnesia sarmientoi Lorentz ex Griseb. (Zygophyllaceae): estudio de base para su inclusión en el apéndice II de la Convención CITES. WWF Paraguay. Lambaré, Paraguay. Pp. 15.

Ministerio de Justicia y Derechos Humanos. Ley 22.344. Buenos Aires. Convenciones Internacionales. $1^{\circ}$ de diciembre 
de 1980. Presidencia de la Nación. URL: servicios.infoleg.gob.ar.

Oliver, C., and B. Larson. 1996. Forest stand dynamics. Editorial Wiley and Sons.

Pinazo, M. A., and N. I. Gasparri. 2003. Cambio estructurales causados por el aprovechamiento selectivo en el Bosque Montano del norte de Salta, Argentina. Ecología Austral 13:160-172.

Piquer-Rodríguez, M., S. Torrella, G. Gavier-Pizarro, J. Volante, D. Somma, R. Ginzburg, and T. Kuemmerle. 2015. Effects of past and future land conversions on forest connectivity in the Argentine Chaco. Landscape Ecol 30:817-833.

R Core Team. 2017. R: A language and environment for statistical computing. R Foundation for Statistical Computing, Vienna, Austria. URL: www.R-project.org.

Sasaki, N., and F. E. Putz. 2009. Critical need for new definitions of "forest" and "forest degradation" in global climate change agreements. Conserv Lett 2:226-232. DOI:10.1111/j.1755-263X.2009.00067.x.

Secretaría de Ambiente y Desarrollo Sustentable. 2013. Resolución 393/13. Exportación de Palo Santo. URL: argentinambiental.com/legislacion/nacional/resolucion-39313-exportacion-palo-santo.

Suzuki, R., and H. Shimidaira. 2006. Pvclust: An R package for assessing the uncertainty in hierarchical clustering. Bioinformatics Applications Note 22:1540-1542.

Waller, T. 2009. Situación de la especie Palo Santo (Bulnesia sarmientoi Lorentz ex Griseb.) en la provincia de Formosa: estudios de sitios de extracción en los departamentos Matacos y Bermejo. Fundación Biodiversidad Argentina. Argentina. Pp. 45. URL: www.academia.edu/2120881/Conservation_of_the_Palo_Santo_tree_Bulnesia_sarmientoi_ Lorentz_ex_Griseb_in_the_South_American_Chaco_Region. 